\title{
ON THE COMPUTATION OF THE MOON'S SPECTROGRAPHIC VELOCITY NEAR FULL MOON
}

The determination of the moon's spectrographic velocity from American Ephemeris data involves the use of the cosine of the angle ( $E$ ) at the earth's center between the sun and moon, and also the product $\sin E \frac{d E}{d t}$. $\quad E$ and the reciprocal of its rate of change, $\frac{d t}{d E}$, are regularly tabulated in the Nautical Almanac except for seven or eight days at full moon, when our satellite is often most favorably situated for spectrographic observation. As a result, the facility of computation of the moon's radial velocity is somewhat impaired, though the problem presents no difficulty. $\operatorname{Cos} E$ is computed directly and $\sin E \frac{d E}{d t}$ is obtained by numerical differentiation of $\cos E$, or more simply by means of differential formulae.

A complete discussion of the five components of velocity to be considered in this case has been given by Professor Campbell.* Components $V_{3}$ and $V_{4}$ only are to be considered here and they will be expressed invariably in kilometers per second. $V_{3}$ is the component of $V_{2}$ (the radial velocity of the moon with reference to the earth's center) in the line joining the sun and moon. $V_{4}$ is the component in this same line of the moon's velocity normal to the radius vector drawn from the moon to the earth. The formulae expressing these quantities are as follows:

$$
\begin{aligned}
& \nabla_{3}=-V_{2} \cos E, \\
& \nabla_{4}=[4.6856] D_{2} \sin E \frac{d E}{d t},
\end{aligned}
$$

where $D_{2}$ is the moon's distance in kilometers from the earth's center and $\frac{d E}{d t}$ is expressed in seconds of are per second of time. $\operatorname{Cos} E$ and $\sin E \frac{d E}{d t}$ may be computed as follows:

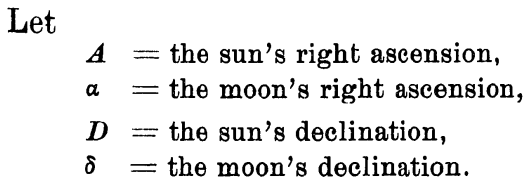

*Astrophysical Journal, 11, 141, 1900.
Then

$$
\begin{aligned}
\operatorname{Cos} E & =\sin \delta \sin D+\cos \delta \cos D \cos (A-a),(1) \\
\sin E \frac{d E}{d t} & =\sin \delta \cos D\left[\cos (A-a) \frac{d \delta}{d t}-\frac{d D}{d t}\right] \\
& +\cos \delta \sin D\left[\cos (A-a) \frac{d D}{d t}-\frac{d \delta}{d t}\right] \\
& +\cos \delta \cos D \sin (A-a) \frac{d(A-a) .}{d t}
\end{aligned}
$$

First Method.-This involves the computation of three values of $\cos E$, and a simple numerical differentation. It is most convenient to compute $\sin E \frac{d E}{d t}$ in radians per hour by numerical differentation of $\cos \boldsymbol{E}$.

Then

$$
\begin{aligned}
& V_{3}=-V_{2} \cos E \\
& V_{4}=[6.4437] D_{2} \sin E \frac{d E}{d t}=278 \times 10^{-i} \times D_{2} \sin E \frac{d E}{d t} .
\end{aligned}
$$

Example.-Determine $\cos E$ and $\sin E_{d t}^{d E}$, in radians per hour, for 1903 November $7^{\mathrm{d}} 19^{\mathrm{h}} 58^{\mathrm{m}}$.

$$
\begin{aligned}
& \text { November } 7 \quad 19^{\mathrm{h}} \quad 20^{\mathrm{h}} \quad 21^{\mathrm{h}} \\
& \text { A } 222^{\circ} 13^{\prime} 57^{\prime \prime} \quad 222^{\circ} 16^{\prime} 27^{\prime \prime} \quad 222^{\circ} 18^{\prime} 57^{\prime \prime} \\
& \begin{array}{llllllllll}
D & -16 & 15 & 14 & -16 & 15 & 59 & -16 & 16 & 43
\end{array} \\
& \begin{array}{lllllllllll}
a & 83 & 54 & 1 & 84 & 31 & 11 & 85 & 8 & 22
\end{array} \\
& \begin{array}{llllllllll}
\delta & 18 & 16 & 14 & 18 & 17 & 01 & 18 & 17 & 41
\end{array} \\
& \begin{array}{llllllllll}
A-a & 138 & 19 & 56 & 137 & 45 & 16 & 137 & 10 & 35
\end{array} \\
& \log \sin \delta \quad 9.49624 \quad 9.49655 \quad 9.49680 \\
& \sin D \quad 9.44699_{\mathrm{n}} \quad 9.44732_{\mathrm{n}} \quad 9.44764_{\mathrm{n}} \\
& \begin{array}{llll}
\cos \delta & 9.97753 & 9.97750 & 9.97747
\end{array} \\
& \cos D \quad 9.98228 \quad 9.98226 \quad 9.98223 \\
& \cos (A-\alpha) \quad 9.87333_{\mathrm{n}} \quad 9.86939_{\mathrm{n}} \quad 9.86537_{\mathrm{n}} \\
& \cos \delta \cos D \cos (A-a) \quad 9.83314_{n} \quad 9.82915_{\mathrm{n}} \quad 9.82507_{\mathrm{n}} \\
& \text { Add log } \quad 0.05264 \quad 0.05317 \quad 0.05370 \\
& \begin{array}{llll}
\sin D \sin \delta & 8.94323_{\mathrm{n}} & 8.94387_{\mathrm{n}} & 8.94444_{\mathrm{n}}
\end{array} \\
& \text { Diff. log } \quad 0.88991 \quad 0.88528 \quad 0.88063 \\
& \begin{array}{cccc}
\log \cos E & 9.88578_{\text {n }} & 9.88232_{\text {n }} & 9.87877_{\text {n }}
\end{array} \\
& \begin{array}{lll}
\cos E-0.76874 & -0.76264 & -0.75643
\end{array} \\
& \frac{d \cos E}{d t}=-\sin E \frac{d E}{d t} \quad+0.00610 \quad+0.00621 \\
& \frac{d^{2} \cos E}{d t^{2}}+0.00011 \\
& {\left[19^{\mathrm{h}} 58^{\mathrm{m}}\right] \cos E \quad-0.763} \\
& {\left[19^{\mathrm{h}} 58^{\mathrm{m}}\right] \sin E \frac{d E}{d t} \quad-0.00615 \text { radians per }} \\
& \text { hour. The } \\
& \text { equivalent of } \\
& \text { - 0"353 per } \\
& \text { second. }
\end{aligned}
$$


Second Method.-Both formulae (1) and (2) are employed. The hourly change in $A$ and $D$, and the velocity in $\alpha$ and $\delta$ per minute are tabulated in the Almanac. It is convenient to reduce all these quantities mentally to seconds of arc per minute or per second of time, varying the factor in $V_{4}$ accordingly. All computations are accomplished with Crelle's Rechentafeln and three-place tables. The formulae for $V_{3}$ and $V_{4}$ when $\frac{d E}{d t}$ is expressed in seconds of are per second are:

$$
\begin{aligned}
& V_{3}=-V_{2} \cos E \\
& V_{4}=[4.6856] D_{2} \sin E \frac{d E}{d t}=485 \times 10^{-8} \times D_{2} \sin E \frac{d E}{d t} .
\end{aligned}
$$

Example.-Determine $\cos E$ and $\sin E \frac{d E}{d t}$ in seconds of are per second of time, for 1903 November $7^{\mathrm{d}} 19^{\mathrm{h}} 58^{\mathrm{m}}$.
1903 November $7^{\mathrm{d}} 19^{\mathrm{b}} 58^{\mathrm{m}}$.

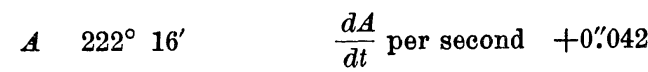

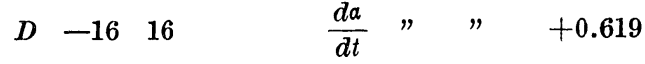

$$
\begin{aligned}
& \text { a } \quad 84 \quad 30 \\
& \text { \& } 18 \quad 17 \\
& \cos (A-a) \frac{d \delta}{d t} \quad-0.009 \\
& \begin{array}{lll}
A-a & 137 \quad 46
\end{array} \\
& \frac{d D}{d t} \text { per second }-0.012 \\
& \sin \delta \quad 0.314 \\
& \frac{d \hat{\delta}}{d t} " \quad " \quad+0.012 \\
& \sin D \quad-0.280 \\
& \cos (A-a) \frac{d D}{d t}+0.009 \\
& \text { Product }-0.088 \\
& \cos \delta \quad+0.960 \\
& \cos D+0.950 \\
& \cos (A-\alpha) \quad-0.740 \\
& \text { Product }-0.675 \\
& \cos E \quad-0.763 \\
& \cos (A-a) \frac{d \delta}{d t}-\frac{d D}{d t}+0.003 \\
& \sin \delta \cos D \quad+0.3 \\
& \text { I Product }+0.001 \\
& \cos (A-a) \frac{d D}{d t}-\frac{d \delta}{d t}-0.003 \\
& \cos \delta \sin D \quad-0.3 \\
& \text { II Product }+0.001 \\
& \frac{d A-d a}{d t}-0.577 \\
& \sin (A-a)+0.672 \\
& \cos \delta \cos D+0.912 \\
& \text { III Product }-0.354 \\
& {[\mathrm{I}+\mathrm{II}+\mathrm{III}] \sin E \frac{d E}{d t}-0 . / 352}
\end{aligned}
$$

Mt. Hamilton, March 11, 1905.

R. H. Curtiss. 